\title{
Design and Test Results of a Transition Radiation Detector for A Fermilab Fixed Target Rare Kaon Decay Experiment
}

\author{
G.E. Graham, N. Solomey, Y.W. Wah, E.D. Zimmerman \\ The Enrico Fermi Institute, The University of Chicago, \\ 5640 S. Ellis Avenue, Chicago, IL 60637, USA \\ M. Daum, Y.B. Hsiung, J.C. Krider, E.J. Ramberg \\ Fermi National Accelerator Laboratory \\ Batavia, IL 60510, USA
}

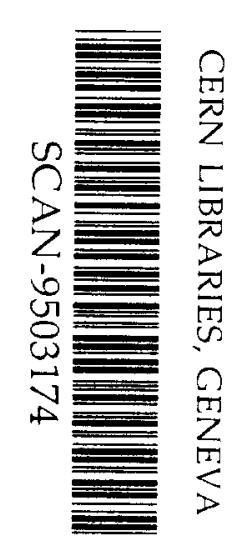

\begin{abstract}
A transition radiation detector with large aperture ( $2.2 \mathrm{~m}$ width $\times 2.2 \mathrm{~m}$ height) has been So) $\operatorname{sich}$ designed and optimized to provide $\pi / \mathrm{e}$ rejection of at least 120:1 in a rare kaon decay experiment (E799) at Fermilab. The momentum range of interest is between 2 and $25 \mathrm{GeV} / \mathrm{c}$. Beam test results with a small prototype wire chamber with different radiators and conventional gas mixtures are shown.
\end{abstract}

Contribution to the 1995 Vienna wire Chamber Conference and accepted to be published in Nuclear Instruments and Methods. 


\section{Introduction}

A transition radiation detector (TRD) with large aperture $(2.2 \mathrm{~m}$ width $\times 2.2 \mathrm{~m}$ height) has been designed to be used in a fixed target rare kaon decay experiment (KTeV-E799) in Fermilab. In particular, this TRD will aid in the search for the direct-CP violating rare decays $\mathrm{K}_{\mathrm{L}} \rightarrow \pi^{0} \mathrm{e}^{+} \mathrm{e}^{-}$and $\mathrm{K}_{\mathrm{L}} \rightarrow \pi^{0} \mathrm{v} v$ by rejecting the dominant $\mathrm{K}_{\mathrm{e} 3}$ background. The momentum range of interest of the $\pi / \mathrm{e}$ rejection is between 2 and $25 \mathrm{GeV} / \mathrm{c}$.

The final KTeV-E799 TRD system will consist of eight identical modules. Each module consists of a dual-plane multi-wire proportional chamber $2.2 \mathrm{~m}(\mathrm{H}) \times 2.2 \mathrm{~m}(\mathrm{~W})$ preceded by a polypropylene fiber mat or foam radiator. The MWPC active gas will be $80 / 20 \mathrm{Xe} / \mathrm{CO}_{2}$. Figure 1 shows the placement of the different components of a single module with the designated wire goemetry.

To optimize the performance of the TRD system for KTeV-E799, there are several experimental constraints and parameters to be attended to. Contraints are: (i) we need to keep the total radiation length of the TRD system to within $10 \%$ of a radiation length; (ii) it needs to be fit into a physical space of less than 2 meter in length; and (iii) the drift time of the chamber needs to be kept under 300 nsec to adaquately suppress beam accidental activities. To obtain highest transmission of the generated TR X-ray spectrum to match with the detection efficiency of the chamber, we studied the performance of different TR materials and compared resolution of three conventional gas mixtures. Our system is further complicated by its large aperture because we need to keep the entrance cathode window very flat $(\sim 100 \mu \mathrm{m})$ so that uniform gain and drift time across the aperture are maintained.

\section{Optimization}

\section{$2.1 X$-ray transmittance}

To compensate for the hydrostatic and gravitational bulging of the stretched $51 \mu \mathrm{m}$ aluminized mylar entrance window due to $\mathrm{Xe}$ in the active chamber volume, a buffer volume about $1 \mathrm{~cm}$ thick was added and filled with a X-ray transparent gas, but with a density closely matched to Xe. A buffer gas, $\mathrm{C}_{2} \mathrm{~F}_{6}$ mixed with $\mathrm{CO}_{2}$, has been tested and was shown to perform satisfactorily. The effect of contamination of $\mathrm{C}_{2} \mathrm{~F}_{6}$ in the active $\mathrm{Xe}$ volume was studied, and found to be acceptable without affecting performance if it was kept below a contamination level of $0.2 \%$. Figure 2 shows the calculated $X$-ray transmittance of the chamber layout as shown in figure 1 . Two data points taken with the triggerable $\mathrm{X}$-ray sources, Mn55 and Zn65, confirmed our expectation. The X-ray transmittance at $8.0 \mathrm{keV}$ was about $72 \%$.

The cathode was a wire plane consisting of $85 \mu \mathrm{m}$ diameter $\mathrm{Be} / \mathrm{Cu}$ wires with $2.5 \mathrm{~mm}$ spacing. The entrance window was kept at a slightly higher voltage than the cathode forming a $1.5 \mathrm{~mm}$ minidrift gap. The anode to cathode gap was $6.5 \mathrm{~mm}$, and the anode was a wire plane consisted of $25 \mu \mathrm{m}$ diameter gold plated tungsten wires with a $5 \mathrm{~mm}$ spacing. 


\subsection{Choice of Gas}

Many gas mixtures were studied for use in the active MWPC regions. The two likely candidates were $80 / 20 \mathrm{Xe} / \mathrm{CO}_{2}$ and $80 / 20 \mathrm{Xe} / \mathrm{CF}_{4}$, and the drift speeds were measured to be $5 \mathrm{~cm} / \mu \mathrm{sec}$ and $6.5 \mathrm{~cm} / \mu \mathrm{sec}$ respectively. However, the energy resolution of the $\mathrm{CF}_{4}$ mixture was worse than the $\mathrm{CO}_{2}$ mixture as shown in figure 3. We choose to use $80 / 20 \mathrm{Xe} / \mathrm{CO}_{2}$ in the final system.

\section{$2.3 T R$ radiator}

A comprehensive study of many different polypropylene transition radiators was carried out at the KEK (Japan National Accelerator Laboratory) electron test beam facility during the summer of 1993. A $1-5 \mathrm{GeV} / \mathrm{c}$ electron beam was produced by internal conversion of synchrotron photons and was nearly $100 \%$ pure. A dual-plane MWPC similar to the one described above but smaller in width and height, was used to detect the TR X-rays. The gas used was $90 / 10 \mathrm{Xe} / \mathrm{CH}_{4}$, and the charge $\mathrm{ADC}$ gate was $300 \mathrm{~ns}$ wide. Each radiator was chosen to have a thickness of one $\mathrm{X}$-ray absorption length at $10 \mathrm{keV}$. This served the purpose of matching the energy of peak TR production approximately with the peak detection efficiency of the MWPC and removed bias from variation in TR production due to variation in the amount of radiator present.

The figure of merit used to rank the performance of each radiator is the percentage of events accompanied by transition radiation. In order to determine this quantity, pulse height spectra were taken with each radiator in place, then replaced with a polypropylene plate with the same radiation length. The effect of the polypropylene plate was to remove bias due to $\delta$-rays produced by the TR radiators. By normalizing the height of the ionization peak of the TR radiator pulse height spectrum to that produced by the corresponding plate, we calculated the figure of merit for each radiator as the excess number of entries. Table 1 below shows the test results.

Table 1 : Tested Radiator Materials with Figures of Merit.

\begin{tabular}{|c|c|c|}
\hline Radiator Material & Figure of Merit 1 & $\begin{array}{c}\text { Absorption Length at } 10 \\
\mathrm{keV}(\mathrm{cm})\end{array}$ \\
\hline LASR Fiber Mat ${ }^{2}$ & $(33.3 \pm 1.6) \%$ & 13.0 \\
\hline Triumph Fiber Mat ${ }^{3}$ & $(30.0 \pm 1.5) \%$ & 6.3 \\
\hline Venus Fiber Mat ${ }^{4}$ & $(22.0 \pm 1.5) \%$ & 5.2 \\
\hline E799-I Fiber Mat 5 & $(28.9 \pm 1.5) \%$ & 10.4 \\
\hline Poly Plank Foam ${ }^{6}\left(2.2 \mathrm{lb} / \mathrm{ft}^{3}\right)$ & $(25.0 \pm 1.7) \%$ & 13.0 \\
\hline Poly Plank Foam $\left(4.0 \mathrm{lb} / \mathrm{ft}^{3}\right)$ & $(17.8 \pm 1.5) \%$ & 7.1 \\
\hline Poly Plank Foam $\left(6.0 \mathrm{lb} / \mathrm{ft}^{3}\right)$ & $(8.3 \pm 1.8) \%$ & 4.8 \\
\hline
\end{tabular}


Poly Plank Foam $\left(9.0 \mathrm{lb} / \mathrm{ft}^{3}\right)$

$$
(7.8 \pm 1.8) \%
$$

Rohacell Foam ${ }^{7}$ ( $31 \mathrm{gm} /$ liter)

$(17.0 \pm 1.9) \%$

10.5

Rohacell Foam (110 gm/liter)

(12.2 \pm 1.7$) \%$

Rohacell Foam ( $170 \mathrm{gm} /$ liter)

$(9.7 \pm 1.9) \%$

2.0

Polypropylene Foil Stack

(32.9 \pm 1.9$) \%$

$0.50(286$ foils at $180 \mu \mathrm{m} /$ foil $)$

\section{CERN Beam Test Results}

The final design of the dual-plane MWPC was tested at a $10 \mathrm{GeV} / \mathrm{c}$ electron test beam facility at CERN in the spring of 1994. It was monitored by two Cerenkov counters and was estimated to be $98.5 \%$ pure. The gas used in the MWPC was $80 / 20 \mathrm{Xe} / \mathrm{CO}_{2}$, and the charge ADC gate was 300 ns wide. As in the KEK beam test of the previous summer, ionization-only spectra were obtained by running with a polypropylene plate in place of the radiator. The pulse height spectrum obtained with the radiator (electron-like) and that with the plate (pion-like) were shown in figure 4 for the upstream MWPC plane only. The TR peak was well separated from the ionization peak.

\section{Projected Performance}

A TRD system consisting of eight modules will be used in $\mathrm{KTeV}$. We use the maximum likelihood method to predict the pion rejection of the final system from the beam test result shown above. The logarithm of the maximum-likelihood of a event generated randomly from the measured pulse height spectra was calculated using the following formula,

$$
\log \mathrm{L}=\Sigma \log \left[\mathrm{P}_{\pi, \mathrm{i}}(\mathrm{E}) / \mathrm{P}_{\mathrm{e}, \mathrm{i}}(\mathrm{E})\right] \quad \text { where } \mathrm{i}=1,2 \ldots 15,16 .
$$

$P_{e, i}(E)$ is the probability for an electron to produce a signal $E$ in plane $i$ and $P_{\pi, i}(E)$ is the probability for a pion to produce a signal $\mathrm{E}$ in plane $\mathrm{i}$. The rejection is then defined as the electron efficiency to pass a cut on $\log \mathrm{L}$ divided by the pion efficiency to pass the same cut.

Because of the discrete nature of the underlying pulse height spectra, further optimization was done by varying bin size of the observed energy spectra for a constant number of events in each distribution and for the scale of the distribution. This optimization ensured the validity of the rejection calculation. We projected for a eight module TRD system, the pion rejection is 40:1 for $90 \%$ electron efficiency if only the upstream plane is used in each of the MWPC. The rejection is 120:1 if both planes are used. Figure 5 shows the calculated rejection as a function of electron efficiency. KTeV will take physics data in 1996.

\section{References}

[1] Error is statistical only. Also this KEK data was taken with the 3mm mini-drift gap turned off (the final design 
is $1.5 \mathrm{~mm}$ wide gap powered on), the projected figure of merit with the final design is substantially higher as shown by the CERN test beam data in figure 4 . The table is intended for relative comparison of different radiator performance only.

[2] S. P. Swordy et. al., Nucl Instr. and Meth. 193 (1982) 591-596. LASR stands for Laboratory for Astrophysics \& Space Research of The University of Chicago. This olefin fiber was manufactured by Hercules Company (type $101,1.8$ denier) and has a diameter of $17 \mu \mathrm{m}$. The mat has a bulk density of $0.04 \mathrm{gm} / \mathrm{cm}^{3}$. We thank S. Swordy and D. Muller for providing the test sample.

[3] This polypropylene fiber was manufactured by Carl Freudenberg, Postfach 1369, D-6940 Weinheim, Germany for TRIUMF in Canada. The fiber diameter is also $17 \mu \mathrm{m}$ woven into a density of $0.1 \mathrm{~g} / \mathrm{cm}^{3}$. We thank $\mathrm{M}$. Vetterli and $\mathrm{A}$. Miller for providing us the sample.

[4] M. Sakuda et. al., Nucl. Instr. and Meth. A311 (1992) 52-77; Nucl. Instr. and Meth. A248 (1986) 379. The mat is irregularly woven from $18 \mu \mathrm{m}$ polypropylene fiber to a density of $0.116 \mathrm{gm} / \mathrm{cm}^{3}$. We thank M. Sakuda for his help.

[5] This fiber was used in the test run of E799 and is very similar to the LASR fiber. The tested mat was made of 16 layer of felt, each 0.25 inches thick and has a density of $9 \mathrm{oz} / \mathrm{yd}^{2}$.

[6] Closed-cell polyethylene foam.

[7] ROHM GmbH, Postfach 4242, Kirschenallee, D-6100 Darmstadt 1, Germany.

Figure Captions:

Fig. 1 Schematics of the radiator and MWPC geometry.

Fig. 2 Transmission of a single TR detector. Curve a) is for the two layer of aluminized mylar foils, b) is for the $1 \mathrm{~cm}$ of buffer gas, and c) is for the wire geometry. Dotted curve is for the absorption of the active Xe gas. Net transmission is shown as the dark solid curve. Two measured points from X-ray sources are in good agreement with the calculated curve.

Fig. 3 Pulse height distributions of $8 \mathrm{keV} \mathrm{X}$-ray for different gas mixtures, solid line is $80 / 20 \mathrm{Xe} / \mathrm{CO}_{2}$, dash line is $80 / 20 \mathrm{Xe} / \mathrm{CH}_{4}$, and dotted line is $80 / 20 \mathrm{Xe} / \mathrm{CF}_{4}$. The chamber operating voltage was adjusted for corresponding gas mixtures so that the 8 keV X-ray peaks always have the same mean ADC counts.

Fig. 4 The ionization spectrum (solid) and the TR plus ionisation spectrum (dashed) for 10 $\mathrm{GeV}$ electrons with the chamber filled with $80 / 20 \mathrm{Xe} / \mathrm{CO}_{2}$ mixture. 
Fig. 5 Calculated $\pi /$ e rejection vs. electron efficiency for a system of eight TRD modules using the measured spectrum from figure 4 . The dashed curve used only the front MWPC plane while the solid curve used both planes. 


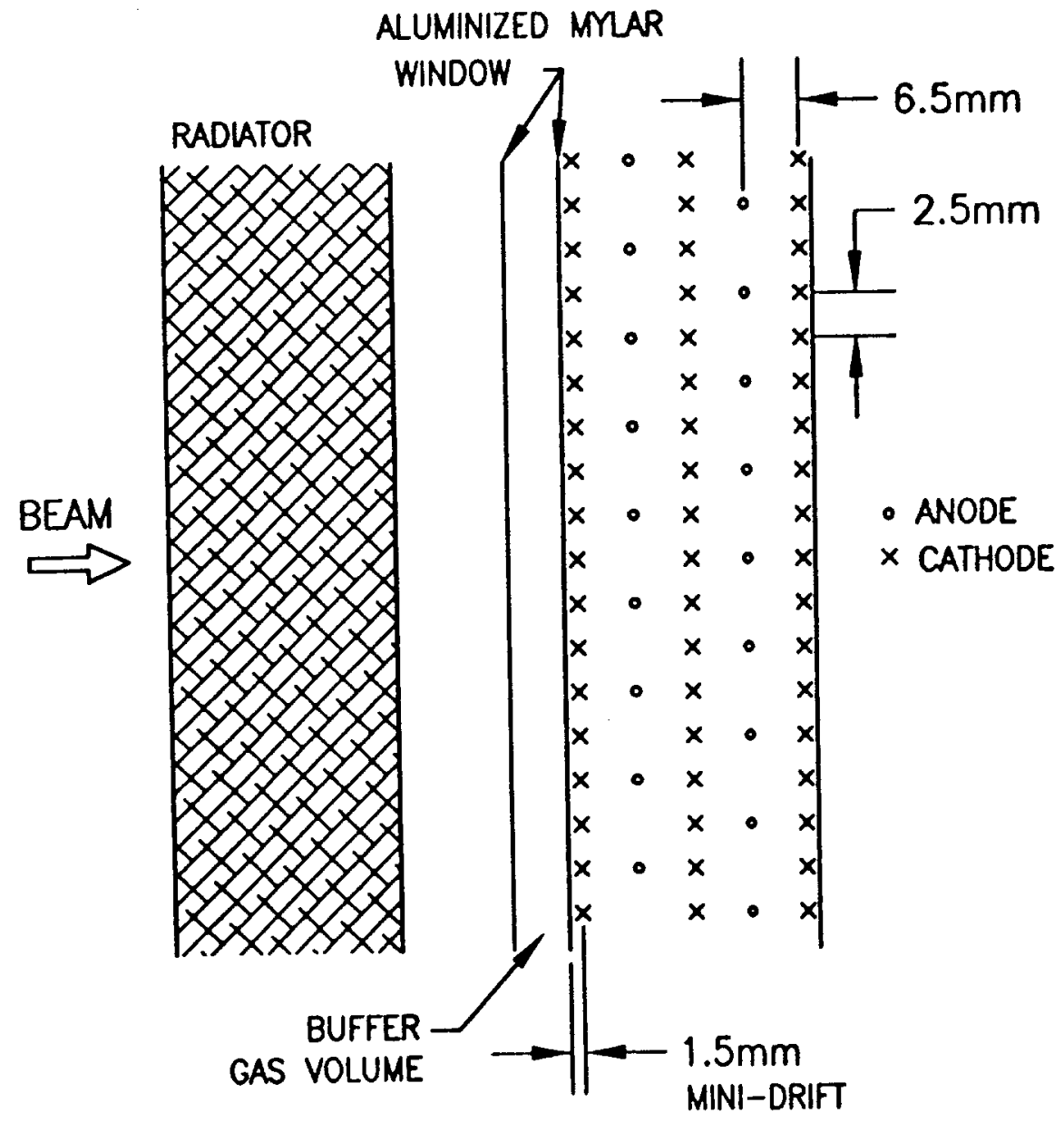

Fig. 1 Schematics of the radiator and MWPC geometry. 


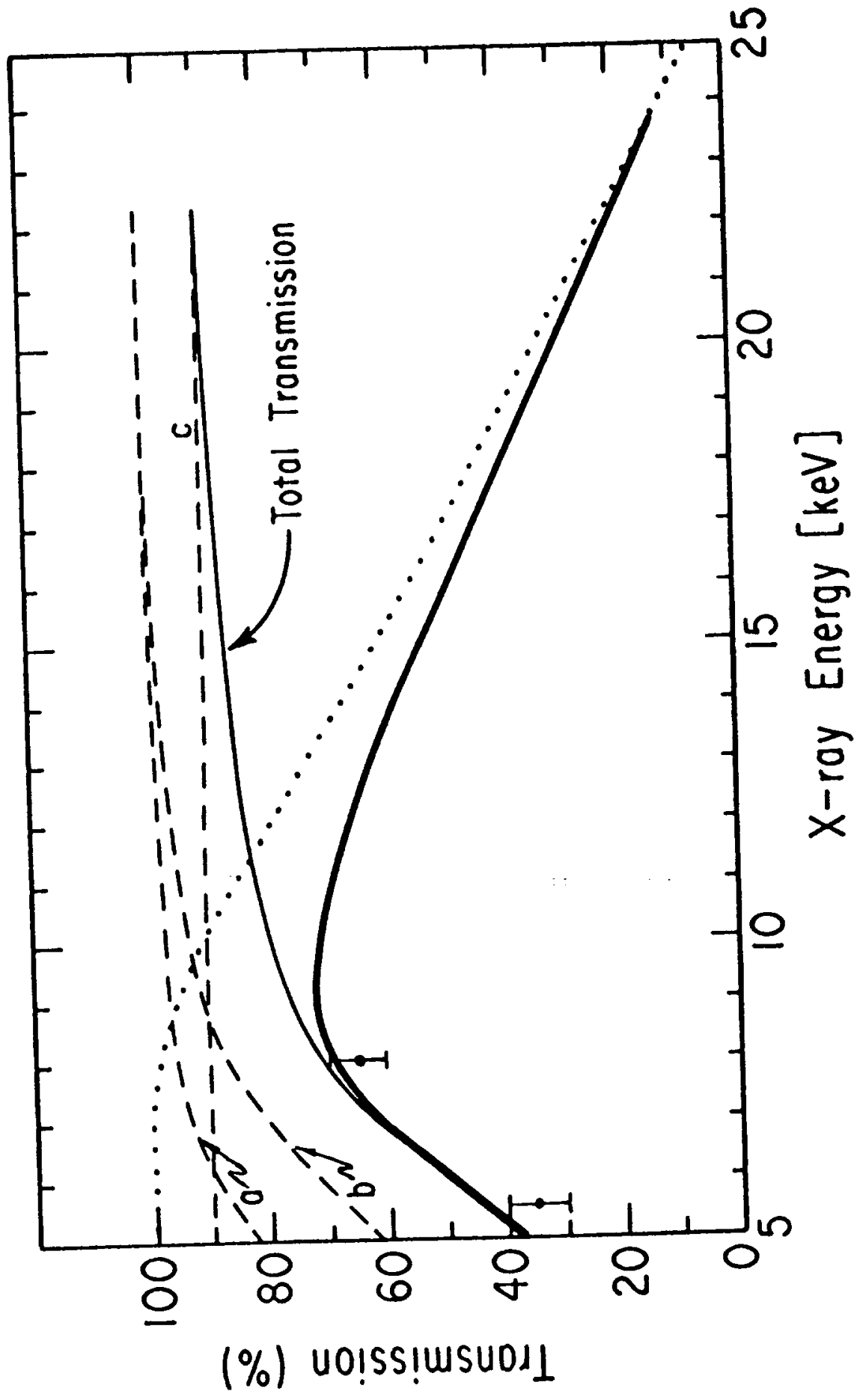

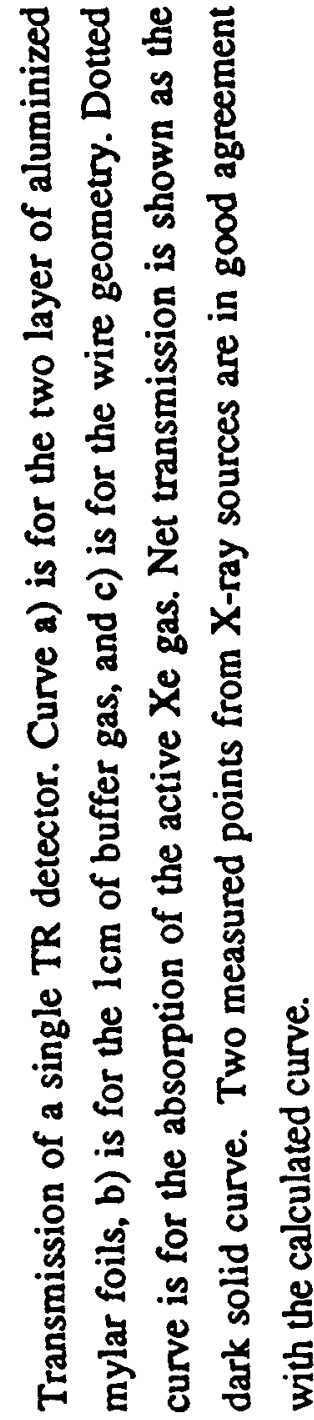

D 
Fig. 3 Pulse height distributions of $8 \mathrm{keV} \mathrm{X}$-ray for different gas mixtures, solid line is $80 / 20 \mathrm{Xe} / \mathrm{CO}_{2}$, dash line is $80 / 20 \mathrm{Xe} / \mathrm{CH}_{4}$, and dotted line is $80 / 20 \mathrm{Xe} / \mathrm{CF}_{4}$. The chamber operating voltage was adjusted for corresponding gas mixtures so that the 8 $\mathrm{keV} \mathrm{X}$-ray peaks always have the same mean ADC counts.

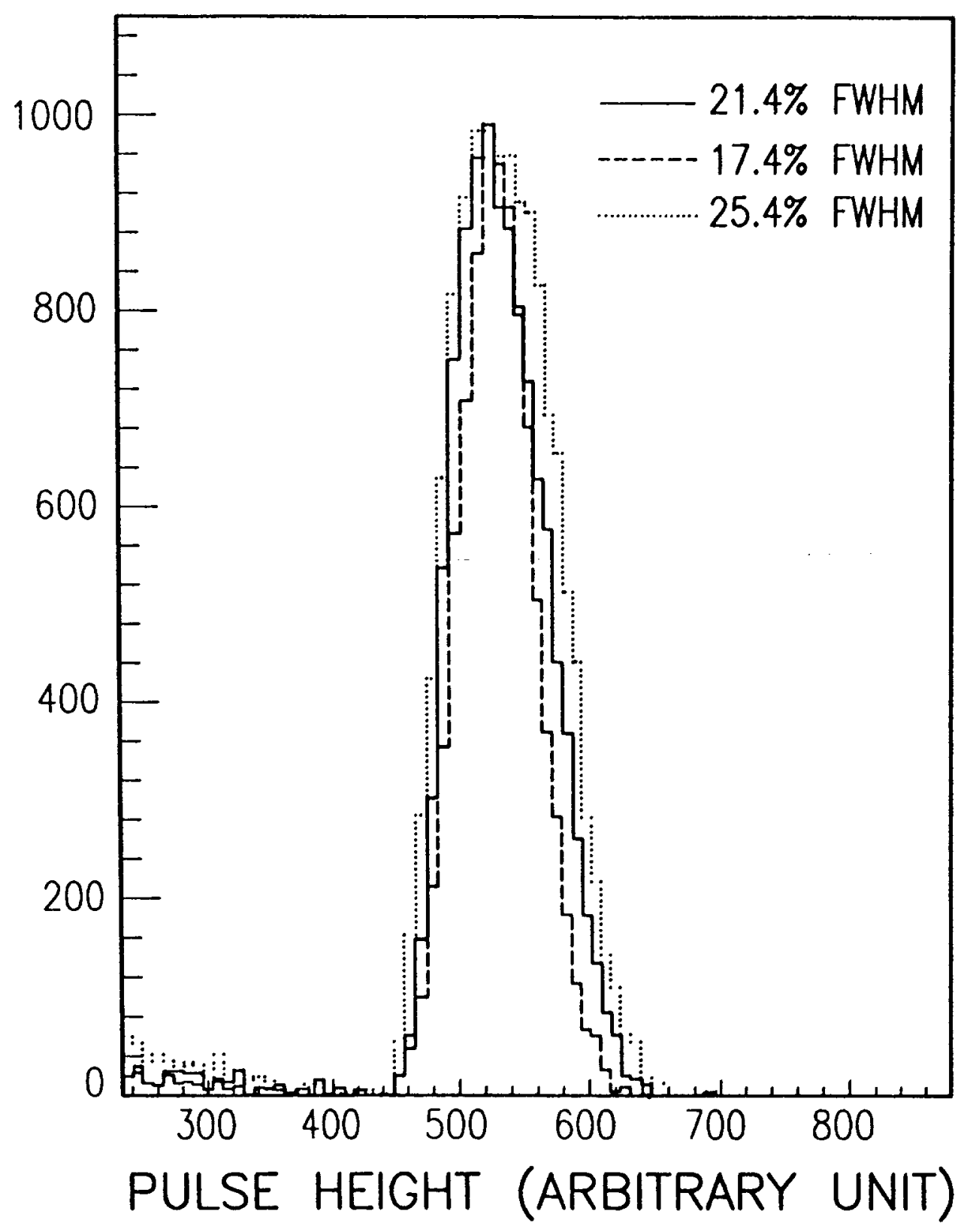


Fig. 4 The ionization spectrum (solid) and the TR plus ionisation spectrum (dashed) for 10 $\mathrm{GeV}$ electrons with the chamber filled with $80 / 20 \mathrm{Xe} / \mathrm{CO}_{2}$ mixture.

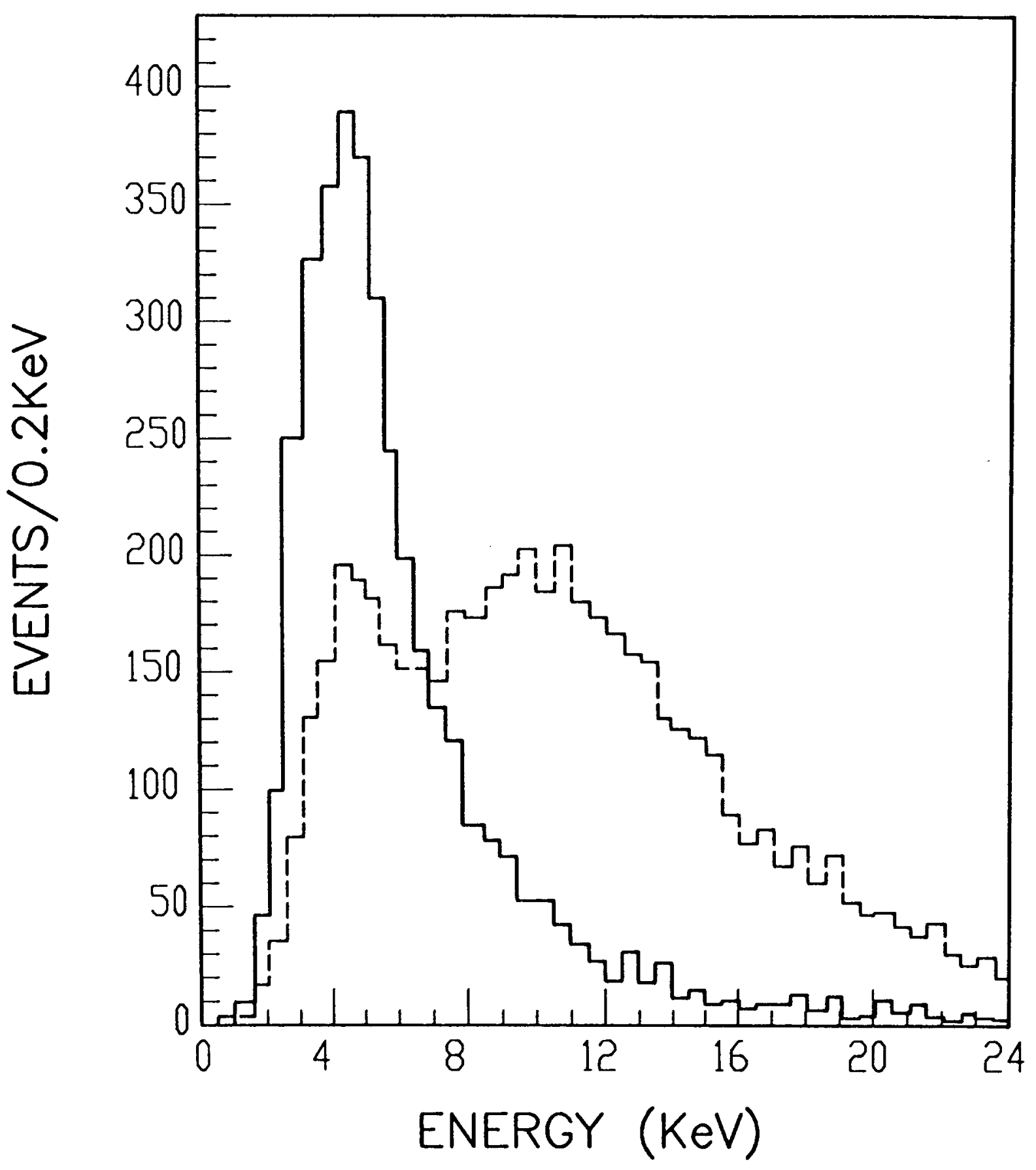




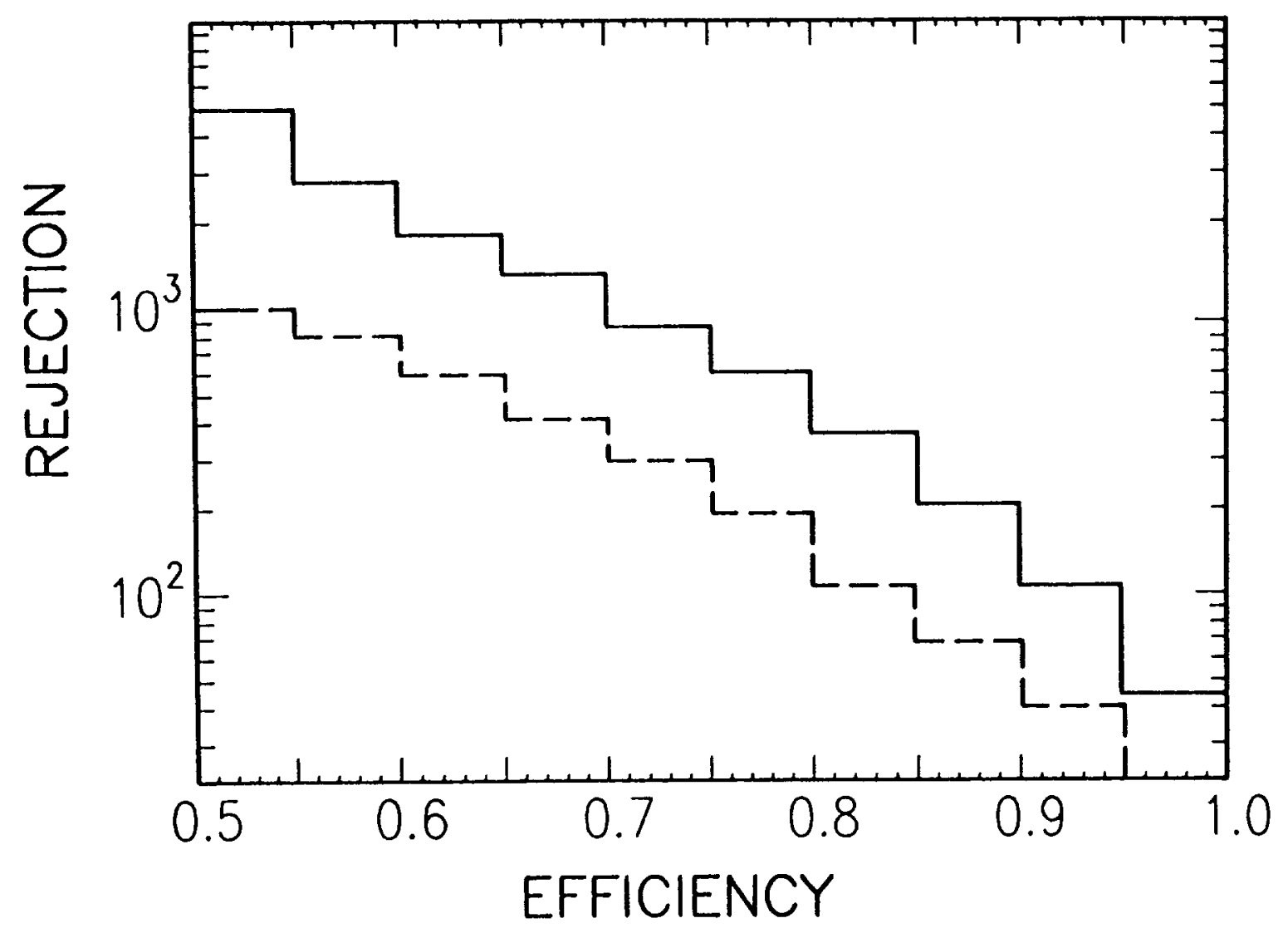

Fig. 5 Calculated $\pi /$ rejection vs. electron efficiency for a system of eight TRD modules using the measured spectrum from figure 4 . The dashed curve used only the front MWPC plane while the solid curve used both planes. 